\title{
Robot-Assisted Laparoscopic Simple Prostatectomy in Men with Bladder Outlet Obstruction: Modified Urinary Reconstruction Leading to Improving Perioperative Outcomes
}

\section{Anup Vora, MD}

Department of Urology, Washington Hospital Center, Georgetown University, Washington, District of Columbia.

E-mail: anupvora@gmail.com

Andrew Harbin, MD

Department of Urology, Washington Hospital Center, Georgetown University, Washington, District of Columbia.

\section{Dan Marchalik, MD}

Department of Urology, Washington Hospital Center, Georgetown University, Washington, District of Columbia.

\section{Hannah Nissim, MD}

Department of Urology, Washington Hospital Center, Georgetown University, Washington, District of Columbia.

\section{Mohan Verghese, MD}

Department of Urology, Washington Hospital Center, Georgetown University, Washington, District of Columbia.

\section{Gaurav Bandi, MD}

Department of Urology, Washington Hospital Center, Georgetown University, Washington, District of Columbia.

\section{Jonathan Hwang, MD}

Department of Urology, Washington Hospital Center, Georgetown University, Washington, District of Columbia.

(c) Mary Ann Liebert, Inc. DOI: 10.1089/vid.2013.0004
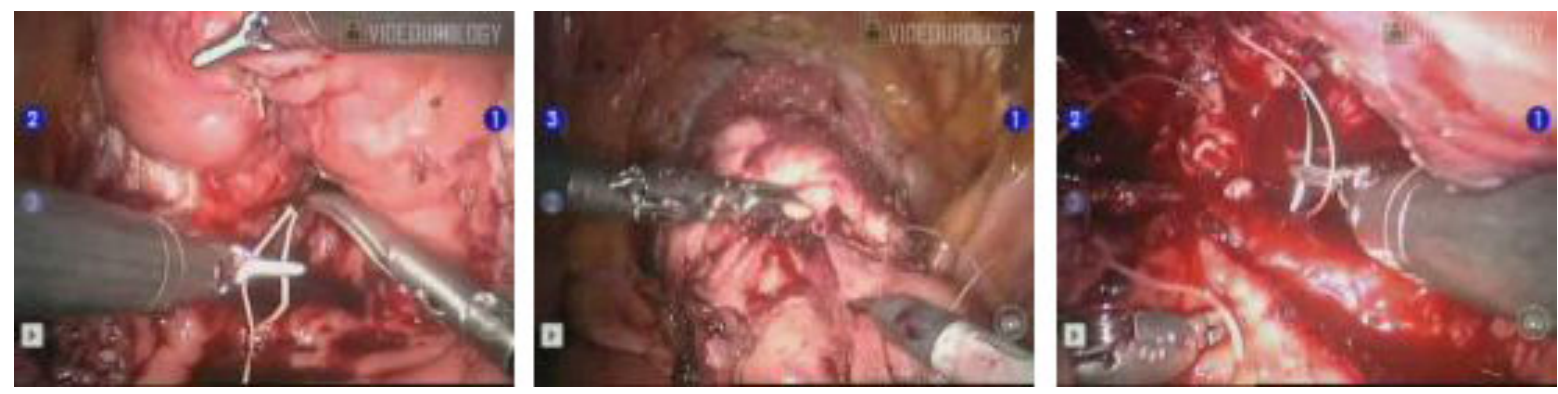

\section{Abstract}

Purpose: Robot-assisted laparoscopic simple prostatectomy (RALSP) is a feasible option in men with markedly enlarged prostates (over $100 \mathrm{~g}$ ) and urinary obstruction. ${ }^{1}$ Since January 2011 , we have modified our surgical method in hope of further enhancing the perioperative outcomes related to RALSP. Herein, we report our initial experience utilizing a circumferential urinary reconstruction technique. 
Methods: A total of nine patients underwent RALSP with a surgical modification for symptomatic benign prostatic hypertrophy (BPH) in 2011. All, but one patient, were in complete urinary retention preoperatively. All RALSP were performed via the suprapubic approach. Upon removal of the obstructing prostate adenomas, a circumferential anastomosis was completed by advancing and approximating the bladder neck to the prostate urethral stump with 8 to 10 interrupted 2-0 vicryl suture, similar to that of the urethral vesical anastomosis during robotic radical prostatectomy. Six patients who underwent a standard RALSP with bladder trigonization only between August 2009 and December 2010 served as the control group.

Results: The two groups were similar in regard to age, BMI, and preoperative prostate size on ultrasonography. No patients in the experimental group required transfusion, suprapubic catheter insertion, or continuous bladder irrigation perioperatively. The control patients were kept with continuous bladder irrigation until urine cleared. Two patients required transfusion of packed red cells due to dropping hematocrit. The mean operative time, pathologic specimen weight, and catheterization time were comparable between the 2 groups, but the average blood loss (100 cc vs. $330 \mathrm{cc}, p<0.05$ ) and hospital stay $(1.4$ days vs. 3.6 days, $p<0.05)$ were significantly shorter in men who underwent RALSP with the surgical modification.

Discussion and Conclusion: Our early experience with the modified RALSP technique (a circumferential anastomosis between the bladder neck and prostate urethra) has been associated with a less intraoperative blood loss and a shorter hospital stay due to early resolution of gross hematuria. In our control group, the length of stay was extended due to the requirement of continuous bladder irrigation and need of transfusion in a third of patients. We feel that our circumferential anastomosis is more hemostatic than previous trigonization-only techniques leading to improved outcomes. One drawback to our technique is increased manipulation of the bladder neck and urethra. While this poses a theoretical risk of bladder neck contracture or stricture, we feel that this risk is minimal, as $<1 \%$ of our robotic radical prostatectomy patients with similar urinary reconstruction have this complication. As other series have reported on modified urethral reconstructions for robotic simple prostatectomies, ${ }^{2}$ larger prospective series with longer follow-up will be necessary to determine the long-term outcomes. Nevertheless, we feel that this reconstruction technique has promise and has shown in our series to have improved perioperative outcomes.

The authors have no conflicts of interest or financial ties to disclose.

Runtime of video: 3 mins 4 secs

\section{Cite this video}

Anup Vora, Andrew Harbin, Dan Marchalik, Hannah Nissim, Mohan Verghese, Gaurav Bandi, Jonathan Hwang, Robot-Assisted Laparoscopic Simple Prostatectomy in Men with Bladder Outlet Obstruction: Modified Urinary Reconstruction Leading to Improving Perioperative Outcomes, J Endourol Part B Videourology. 2013, DOI: 10.1089/vid.2013.0004.

\section{References}

1. Vora A, Mittal S, Hwang J, Bandi G. Robot-assisted simple prostatectomy: Multi-institutional outcomes for glands larger than 100 grams. J Endourol 2012;26:499-502.

2. Coelho RF, Chauhan S, Sivaraman A, Palmer KJ, Orvieto MA, Rocco B, Coughlin G, Patel VR. Modified technique of robotic-assisted simple prostatectomy: Advantages of a vesico-urethral anastomosis. BJU Int 2012;109:426-433.

Original Publication Date: 2013 